\title{
Philosophiques
}

\section{La question des attitudes propositionnelles et les limites de la sémantique}

\section{François Lepage}

Volume 15, numéro 1, printemps 1988

URI : https://id.erudit.org/iderudit/027034ar

DOI : https://doi.org/10.7202/027034ar

Aller au sommaire du numéro

\section{Éditeur(s)}

Société de philosophie du Québec

ISSN

0316-2923 (imprimé)

1492-1391 (numérique)

Découvrir la revue

Citer cet article

Lepage, F. (1988). La question des attitudes propositionnelles et les limites de la sémantique. Philosophiques, 15(1), 59-74. https://doi.org/10.7202/027034ar
Résumé de l'article

Le but de cette intervention est tout d'abord de caractériser un concept de sémantique d'un point de vue suffisamment général pour que l'on puisse l'interpréter comme celui de sémantique universelle. Dans un deuxième temps, il sera question de la caractérisation des contextes extensionnels et un critère général d'identification de tels contextes sera proposé. La thèse suivante, assez surprenante, sera avancée : selon ce critère, les contextes d'attitudes propositionnelles sont extensionnels. La solution que l'on proposera pour sortir de cette situation apparemment paradoxale sera justement de l'interpréter comme un indice du caractère non sémantique du problème des attitudes propositionnelles. Finalement, une voie de solution au puzzle de Kripke sera proposée.
Tous droits réservés (C) Société de philosophie du Québec, 1988
Ce document est protégé par la loi sur le droit d'auteur. L'utilisation des services d'Érudit (y compris la reproduction) est assujettie à sa politique d'utilisation que vous pouvez consulter en ligne.

https://apropos.erudit.org/fr/usagers/politique-dutilisation/ 


\title{
LA QUESTION DES ATTITUDES PROPOSITIONNELLES ET LES LIMITES DE LA SÉMANTIQUE
}

\author{
par François Lepage
}

\begin{abstract}
RÉSUMÉ. Le but de cette intervention est tout d'abord de caractériser un concept de sémantique d'un point de vue suffisamment général pour que l'on puisse l'interpréter comme celui de sémantique universelle. Dans un deuxième temps, il sera question de la caractérisation des contextes extensionnels et un critère général d'identification de tels contextes sera proposé. La thèse suivante, assez surprenante, sera avancée : selon ce critère, les contextes d'attitudes propositionnelles sont extensionnels. La solution que l'on proposera pour sortir de cette situation apparemment paradoxale sera justement de l'interpréter comme un indice du caractère non sémantique du problème des attitudes propositionnelles. Finalement, une voie de solution au puzzle de Kripke sera proposée.
\end{abstract}

\begin{abstract}
The aim of this paper is first to characterize a concept of semantics from such a general point of view that it could be considered as the concept of universal semantics. Extensional contexts will then be characterized and a general identification criterion for such contexts will be given. The following rather surprising thesis will be put forward: According to this criterion, propositional attitude contexts are extensional. The proposed solution to this seemingly paradoxical situation will be to consider propositional attitude contexts as non semantic. Finally, a way out to Kripke's puzzle is propounded.
\end{abstract}

La thèse principale que je vais soutenir dans la présente intervention est simple et radicale: tout en donnant un sens suffisamment précis et raisonnablement englobant au concept de sémantique, je vais tenter de montrer que la solution du problème des attitudes propositionnelles exige des ressources qui débordent ce champ conceptuel, que l'explication même de la nature des 
attitudes propositionnelles fait intervenir des notions qui appartiennent indiscutablement à la pragmatique.

Dans un premier temps, ma tâche consistera donc à présenter ce cadre qui a la prétention d'être suffisamment précis et raisonnablement englobant. Ce cadre, que pour des raisons de brièveté j'appellerai cadre standard, est celui de la théorie des modèles à laquelle on adjoint l'appareillage spécifique de la logique intensionnelle ${ }^{1}$. Dans un deuxième temps, j'essaierai de montrer en quoi ce cadre est trop limité pour pouvoir fournir une explication satisfaisante au problème des attitudes propositionnelles. Finalement, je terminerai en disant quelques mots sur le puzzle de Kripke ${ }^{2}$ et montrerai comment les leçons tirées des réflexions précédentes suggèrent une voie de solution simple, élégante et naturelle à ce fameux puzzle.

\section{LE CADRE STANDARD}

Utiliser le cadre théorique de la théorie des modèles pour tenter d'expliquer un phénomène de signification dans une langue naturelle, c'est mettre de l'avant quelques postulats d'ordre méthodologique et ontologique. Je n'ai pas l'intention d'en faire ici un inventaire exhaustif et je me contenterai de mettre l'accent sur les deux postulats qui me semblent les plus importants. Le premier, fortement lié à la trichotomie classique syntaxe/sémantique/ pragmatique, affirme l'autonomie de la sémantique. On pourrait le

1. Il n'est pas possible de présenter ici ce cadre dans toute sa généralité. Je ne peux que renvoyer le lecteur au texte maintenant classique de Richard MONTAGUE, «Universal Grammar », in Formal Philosophy: Selected Papers of Richard Montague, R. THOMASON (ed.), New Haven and London: Yale University Press, 1974. Cet ouvrage comprend également deux articles un peu plus anciens de MONTAGUE, à savoir «Intensional Logic » et « Pragmatics and Intensional Logic ». Tous ces textes sont relativement difficiles car ils utilisent des ressources logico-mathématiques assez avancées. Le texte de David Lewis, "General Semantics" in Semantics of Natural Language, Davidson, D. et Harman, G. (ed.), Dordrecht : Reidel, 1972, est beaucoup plus accessible. En français, il n'existe que peu de textes. On pourra consulter l'article de Paul GOCHET « La sémantique récursive de Davidson er Montague » in Penser les les mathématiques, Paris : Seuil, 1982 ainsi que mon article « Logique intensionnelle et langues naturelles », in Protée, 12, $\mathrm{n}^{0} 3$, $1984,23-31$.

2. Je supposerai connu du lecteur le fameux puzzle que je ne rappellerai pas ici. Le lecteur devrait lire ou relire S. KRIPKE, "A Puzzle About Belief», in Meaning and Use, Margalit, A. (ed.), Dordrecht : Reidel, 1979, 239-283. 
résumer en disant que les expressions des langues naturelles ont une signification objective, indépendante des utilisateurs (j'utilise l'expression «signification d'une expression" pour désigner ce à quoi renvoie cette expression, sans lui attribuer, pour l'instant, un sens technique).

Cette prise de position en faveur de l'indépendance de la signification des expressions n'est en aucun cas un argument contre le caractère conventionnel de la signification. Bien au contraire, prêter aux expressions une signification conventionnelle n'est qu'une manière, entre autres, de fixer le contenu sémantique objectif de ces expressions. Il est fort possible qu'un agent ait, à un moment ou un autre de son existence, à participer au processus qui établit la convention mais cela n'a rien à voir avec la notion de sémantique elle-même. Par exemple, le prêtre qui baptise un enfant fixe la valeur (une des valeurs) du nom propre et une fois que le baptême a eu lieu, il ne dépend plus des intentions ni du prêtre ni de qui que ce soit d'autre que ce nom soit celui de l'enfant.

Il s'agit là bien sûr d'un cas limite et il est bien évident que les processus par lesquels là où les conventions s'établissent sont dans la majorité des cas extrêmement complexes, souvent sousdéterminants ou ambigus. Prenons le cas très simple du mot "pluie». Il n'est pas difficile d'imaginer des cas où les locuteurs dont on peut raisonnablement supposer qu'ils adhèrent à la convention régissant le contenu sémantique de ce mot, seraient en désaccord pour déterminer si telle ou telle entité est de la pluie ou non (cas de bruine, de neige fondante, etc.). La sous-détermination du sens est un problème qu'il faut prendre en considération nous verrons qu'il n'est pas étranger à notre problématique mais n'affecte pas la thèse de l'indépendance des valeurs sémantiques qui porte exclusivement sur l'autonomie du champ conceptuel de la sémantique.

Ainsi, selon le premier postulat, les mots de notre langue par exemple, auraient une signification objective indépendante des utilisateurs que nous sommes. Exterminons les Québécois jusqu'au dernier et quelque part dans quelque ciel platonicien, restera la signification du mot « poutine». Difficile à avaler. Même le plus québécois des philosophes réalistes n'oserait soutenir une telle thèse. Alors, doit-on au contraire considérer les significations 
comme des entités intentionnelles? Comment dans ce cas expliquer la communication? Ces questions, bien que fondamentales, ne m'intéressent pas ici. Mon problème est plutôt celui de décrire une certaine notion de sémantique, notion qui contient en elle-même l'idée d'externalité de la signification, et de convaincre que le cadre conceptuel et méthodologique qui se déploie à cette occasion rend compte de façon correcte d'une intuition largement partagée. Qu'un tel cadre conceptuel puisse se révéler utile pour expliquer les phénomènes de communication humaine est une chose que l'histoire tranchera; qu' un tel cadre conceptuel ait une richesse et une autonomie suffisante pour constituer une discipline, l'histoire a tranché.

De ce point de vue, la tâche principale de la sémantique est la caractérisation de la manière dont les systèmes symboliques sont générateurs de significations complexes à partir de significations élémentaires et les limites du cadre standard sont justement celles de cette conception. On peut résumer cela en disant que pour les langages qui nous intéressent, il existe une fonction interprétation $f$ qui à chaque expression d'une langue $L$ associe une signification (appelons S l'ensemble des significations). Symboliquement

$$
\mathrm{f}: \mathrm{L} \rightarrow \mathrm{S}
$$

Quelle que soit l'origine de cette fonction, quelle que soit la nature des problèmes relatifs à l'accession des agents cognitifs à cette fonction, nous postulons son existence et en faisons la pierre angulaire de notre approche. L'objectivité des significations trouve ainsi son expression dans la contrainte que pour la description de $f$ n'intervient aucun concept idiosyncratique ou pouvant être soupçonné de l'être, telles les notions d'intention, de compétence et bien d'autres qui font intervenir les agents.

Le deuxième postulat a pour but de rendre compte de la complexité finitaire des langues naturelles qui ont pourtant un pouvoir expressif illimité. Ce second postulat impose donc deux restrictions relativement indépendantes. La première, bien connue, concerne le respect de la thèse de la compositionnalité du sens : la valeur sémantique d'une expression complexe résulte d'une combinatoire finie à partir des valeurs sémantiques d'expressions de base, elles-mêmes en nombre fini. 
La seconde restriction concerne le caractère finitaire des valeurs sémantiques des expressions de base elles-mêmes. Il est plus difficile de donner une formulation précise à cette deuxième restriction. Encore une fois, je ne peux que faire appel à l'intuition : les significations de base ne doivent pas être d'une complexité indéfinie. Cela ne signifie pas, bien sûr, que toute les expressions renvoient à des entités finies mais à des entités d'une complexité finitaire. Ces deux restrictions reposent sur des considérations intuitives et reçoivent leur crédibilité de ce que nous devons pouvoir rendre compte, au moins en théorie, du fait que les langues naturelles sont apprises par des agents finis.

\section{UNE SÉMANTIQUE UNIVERSELLE}

Le type d'interprétation le plus simple que l'on puisse imaginer et qui soit exprimable à l'intérieur de ce cadre est ce que l'on désigne habituellement sous le nom d'interprétation dénotationnelle. Dans ce genre d'interprétation, un nom propre dénote tout simplement l'objet dont il est un nom propre et un prédicat la classe des objets auxquels le prédicat s'applique. Les énoncés, eux, dénotent tout simplement des valeurs de vérité. Si le langage n'est pas du premier ordre, on répète le processus en introduisant toute une hiérarchie de classes de classes par le mécanisme de récursion bien connu.

De nombreux arguments ont été avancés pour souligner l'insuffisance de ce genre de structure à rendre compte de manière intuitivement adéquate des conditions de vérité de certains énoncés. L'exemple probablement le moins discutable concerne les conditions de vérité des énoncés comportant des opérateurs modaux. L'interprétation "naturelle » d'énoncés du genre «Il est possible que $\mathrm{p}$ » semble commander que leurs conditions de vérité dépendent non seulement de ce qui de fait est, donc des dénotations des expressions, mais de ce qu'elles pourraient bien être. Les langues naturelles sont d'ailleurs remplies d'opérateurs de ce genre et d'autres qui, à première vue, semblent encore plus réfractaires à une interprétation dénotationnelle. Par exemple, des énoncés du genre «Il est démontrable en mathématiques que $\mathrm{p}$ », et enfin les énoncés auxquels nous nous intéressons plus spécifiquement ici, les énoncés dits 
d'attitudes propositionnelles «Pierre sait que $\mathrm{p}$ » ou «Pierre croit que $\mathrm{p}$ » ou encore «Pierre désire que $\mathrm{p}$ ».

Dire que les interprétations de type dénotationnel ne peuvent pas rendre compte de ce genre de problème de manière intuitivement adéquate ne suffit pas. Il faut pouvoir exprimer cette insuffisance à l'intérieur du cadre théorique qui est le nôtre en faisant ressortir quelque inconsistance ou du moins quelque incongruité. Prenons l'exemple le plus simple, celui des opérateurs modaux. Selon le deuxième postulat, celui de la compositionnalité du sens, deux expressions qui possèdent la même signification sont substituables partout salva significatione. Dans une interprétation de type dénotationnel, cela s'exprime par le fait que deux expressions de même dénotation sont substituables salva veritate car les significations des expressions sont leurs dénotations et la dénotation d'un énoncé, suivant Frege, est sa valeur de vérité. Remarquons que c'est une conséquence analytique de l'adoption du second postulat : les relations d'intersubstituabilité ne peuvent séparer les expressions en classes d'équivalence plus fines que celles déterminées par la relation d'identité de contenu sémantique. En particulier, puisque les énoncés dénotent une valeur de vérité, deux énoncés de même valeur de vérité devraient, en principe, être substituables partout. Supposons qu'en ce moment il ne pleuve pas. L'énoncé «Il pleut » a la même valeur de vérité que l'énoncé « $2+2=5$ ». Or, nous ne voulons pas, intuitivement, que l'énoncé «Il est possible qu'il pleuve » ait la même valeur de vérité que «Il est possible que $2+2=5 »$. Mais il y a plus. Accepter cette conséquence intuitivement réductrice, serait accepter que d'une façon générale, l'opérateur de possibilité, tout comme celui de nécessité, soit sémantiquement tout à fait inoffensif: il nous est loisible de le mettre devant n'importe quel énoncé ou, au contraire, de l'effacer devant n'importe quel énoncé sans modifier la valeur de vérité de l'expression ainsi obtenue. Bref, les expressions «possible » et «nécessaire » sont vides et la langue française comporte au moins deux mots inutiles.

Pour contourner ce genre de difficultés, une approche bien précise s'impose presque naturellement comme voie de solution : raffinons les significations des expressions jusqu'à ce que nous puissions rendre compte des distinctions exigées par l'intuition. Mais ce raffinement devra-t-il se faire de manière $a d b o c$, au coup 
par coup ? Oui et non. Chaque cas devra recevoir un traitement spécifique dans un cadre absolument universel que je vais maintenant présenter en quelques mots.

La dénotation comme signification d'une expression ne semble pas un objet assez fin pour que l'identité de dénotation soit la relation d'équivalence la plus fine que nous soyons en droit d'attendre. Intuitivement, il semble qu'au-delà de sa dénotation, une expression (surtout si elle est complexe) possède un mode de donation objectif de sa dénotation. Ce mode de donation est constant bien que la dénotation, elle, puisse varier selon les circonstances. L'exemple classique est celui des descriptions définies : au-delà de sa signification constante, l'expression «le président des États-Unis », change régulièrement de dénotation.

Peut-on représenter ce type de phénomène à l'intérieur de notre approche? Bien sûr. Il s'agit tout simplement de remplacer la dénotation d'une expression comme signification par une fonction, ce mot étant utilisé ici au sens technique. Revenons à notre exemple. La différence entre « $2+2=5$ » et «Il pleut » est que le second énoncé dénote le vrai ou le faux en vertu de certaines circonstances alors que ce n'est pas le cas pour le premier qui reste vrai dans toutes les circonstances. (J'appelle circonstance la collection des traits pertinents pour la compréhension, quelle que soit leur nature). C'est justement ce que nous cherchions à exprimer lorsque nous remarquions que la valeur de vérité de «Il est possible qu'il pleuve » ne dépend pas uniquement des circonstances qui font que «Il pleut» est vrai ou non. La signification de «Il pleut» sera donc cette fonction qui prend pour argument ces circonstances et pour valeur le vrai ou le faux selon que, dans ces circonstances, il pleut ou non. La signification d'un nom propre est cette fonction qui à chaque circonstance associe l'objet dont il est le nom propre; la signification d'un prédicat est cette fonction qui à chaque circonstance associe la classe des objets qui tombent sous le prédicat dans cette circonstance; la signification d'un énoncé est cette fonction qui à chaque circonstance associe la valeur de vérité de cet énoncé dans ces circonstances. Si l'énoncé est de la forme $\mathbf{P}(\mathbf{a})$, c'est-à-dire qu'il est de la forme sujet-prédicat, il est très facile de définir cette fonction de sorte qu'elle respecte le principe de compositionnalité : à chaque circonstance elle associe la valeur de vérité vrai si et seulement si dans cette circonstance l'objet 
associé à a dans cette circonstance est dans la classe associée à $\mathbf{P}$ dans la même circonstance. Appelons intensions ces fonctions et extensions les valeurs de ces fonctions, soient les anciennes dénotations, et monde possible la partie des circonstances d'évaluation qui sont indépendantes du fait de l'énonciation ${ }^{3}$.

Nous sommes maintenant en mesure de décrire la signification de "possible»: c'est simplement cette fonction qui à chaque circonstance associe un ensemble de mondes, que l'on peut interpréter comme étant justement l'ensemble des autres mondes possibles. L'énoncé «il est possible que $\mathrm{p}$ » sera vrai dans un monde si et seulement si la signification de $\mathrm{p}$ prend la valeur vrai dans au moins un des mondes de l'ensemble des mondes attachés à «possible» dans cette circonstance.

Les significations des énoncés sont donc des fonctions de circonstances d'évaluation dans valeur de vérité. Que peut-on ou doit-on mettre dans ces circonstances d'évaluation? N'importe quoi à la stricte condition de ne pas violer les postulats de départ. En particulier, on ne saurait mettre dans ces circonstances, pour contourner le postulat d'objectivité, quelque chose qui tiendrait compte des intentions du locuteur, ou plus généralement de l'utilisateur de l'expression : cela supposerait que pour comprendre la signification d'une expression, l'on doive manipuler un objet indéfiniment complexe. En effet, dans ce cas précis, cette signification comprendrait une fonction ayant pour domaine quelque chose comme l'ensemble des intentions des locuteurs, ce qui me semble un bel exemple d'objet indéfiniment complexe.

Ce raffinement des significations en intension s'est avéré d'une grande utilité et intuitivement adéquat pour rendre compte des notions modales comme les opérateurs «possible» et «nécessaire» ou encore les opérateurs temporaux. Mais avant d'aborder la question des attitudes propositionnelles, nous devons une fois de plus affiner nos instruments.

3. Le lecteur aura compris que je ne veux pas ici m'engager sur le délicat terrain de la nature des contextes d'énonciation qui soulève de nombreux problèmes. 


\section{QU'EST-CE QU'UN CONTEXTE EXTENSIONNEL?}

Admettons donc que les significations soient des intensions. Enrichissons notre ontologie non seulement de ces intensions, mais de tout ce que l'on peut définir à partir de ces entités par les moyens ensemblistes bien connus (classes d'intensions, intensions d'intension, etc.). Comment, maintenant, pouvons-nous tester nos hypothèses sur le caractère intensionnel de telle ou telle expression d'un langage? Nous pouvons d'abord utiliser les opérateurs déjà identifiés à l'intérieur des langues naturelles, comme par exemple l'expression " possible ». En effet, si nous adoptons l'interprétation donnée ci-haut de l'expression «possible » (qui semble impliquer que tous les mondes sont mutuellement compossibles, bien que cette interprétation ne s'impose pas comme la seule acceptable), nous sommes en quelque sorte engagés à ce que si « Il est possible que $\mathrm{p}$ » est vrai, alors «Il est possible que $\mathrm{p}$ soit possible » le soit aussi. Il faut remarquer que dans tous les cas, l'intuition demeure le juge de dernière instance pour décider de la valeur de l'explication de la valeur sémantique d'une expression.

Certains de ces opérateurs, qui se révèlent très utiles, s'imposent de façon assez naturelle. Par exemple l'opérateur "maintenant » dont le rôle est en quelque sorte de bloquer la composante temporelle des circonstances de l'évaluation à sa valeur actuelle. En effet, s'il pleut maintenant, alors il a toujours été le cas qu'il pleut maintenant comme il sera toujours le cas qu'il pleut maintenant.

Les instruments que nous venons de présenter permettent de décrire ce genre de situation de façon simple et élégante. Il suffit de supposer qu'à « maintenant » correspond un opérateur sur intensions d'énoncés. "Maintenant» transforme une fonction donnée de circonstances dans valeur de vérité en une autre fonction de circonstances dans valeur de vérité. Symboliquement, si l'on note $\mathrm{C}$ l'ensemble des circonstances et $c_{\mathrm{e}}$ les circonstances de l'évaluation et $\mathrm{x}$ une intension d'énoncé quelconque

$$
f(\ll \text { maintenant })\left(c_{e}\right) \varepsilon\left(\{\text { vrai, faux }\}^{C}\right)
$$

$f($ «aintenant $)\left(c_{e}\right)(x)$ sera cette intension d'énoncé qui dans $n$ 'importe quelle circonstance donne la valeur que $\mathbf{x}$ prendrait dans une circonstance qui diffère au plus de la circonstance d'évaluation 
par le fait que la composante temporelle est la composante actuelle ${ }^{4}$.

Nous pouvons également nous donner de nouveaux instruments dont la présence dans les langues naturelles est extrêmement douteuse. Par exemple l'opérateur " " » où encore l'opérateur " " " que l'on pourrait paraphraser respectivement par «l'intension de» et par «l'extension de». Ces deux opérateurs sont des cas limites. De la même façon, il nous est loisible non seulement de rechercher, mais de développer toute une panoplie d'opérateurs intermédiaires.

On pourrait critiquer la justesse de cette façon de procéder. Je n'y vois pour ma part aucun problème à la condition de toujours demeurer cohérent. Ce n'est qu'à l'application que l'on pourra juger de la pertinence de la construction. Il me semble, par exemple, tout à fait légitime de dire que l'énoncé « $\square$ `p ", que l'on pourrait paraphraser par «Il est nécessaire que $\mathrm{p}$ dans ces circonstances d'évaluation » est vrai si " $\mathrm{p}$ » est vrai dans ces circonstances si l'on accorde aux expressions " circonstances » « nécessaire », etc. le même sens que celui qui leur a été accordé jusqu'ici. On pourrait à loisir multiplier les exemples.

Ces opérateurs qui, comme « maintenant», bloquent en quelque sorte les intensions des expressions sur leur valeur actuelle, vont nous être très utiles. Appelons projecteurs de tels opérateurs. Formellement, si $\mathbf{x}$ est une composante de circonstances d'évaluation, un projecteur -x (que je noterai $\downarrow_{x}$ ), c'est-à-dire un projecteur sur la dimension $x$, est un opérateur tel que si $\left(c_{1}, \ldots, c_{x a}, \ldots, c_{n}\right)$ sont les circonstances de l'évaluation ( $c_{\mathbf{x a}}$ pour valeur actuelle de la composante $c_{\mathbf{x}}$ ) et $\mathbf{A}$ une expression de la bonne catégorie (soit une expression dont la valeur en chaque circonstance est une intension)

$$
\begin{aligned}
& f\left(\downarrow_{x} A\right)\left[\left(c_{1}, \ldots, c_{x a}, \ldots, c_{n}\right)\right]\left(c_{1}^{\prime}, \ldots c^{\prime}, \ldots, c^{\prime}{ }_{n}\right)= \\
& f(A)\left[\left(c_{1}, \ldots, c_{x a}, \ldots, c_{n}\right)\right]\left(c_{1}^{\prime}, \ldots, c_{x a}, \ldots, c_{n}^{\prime}\right)
\end{aligned}
$$

$\left(\left(c_{1}^{\prime}, \ldots, c_{x}^{\prime}, \ldots, c_{n}^{\prime}\right)\right.$ désigne ici une circonstance quelconque).

Utilisant ce formalisme, nous pouvons décrire avec précision ce qu'est un opérateur extensionnel. Un opérateur $\mathbf{O}$ est extensionnel

4. Pour désigner l'ensemble des foncrions de domaine $\mathbf{Y}$ et de codomaine $\mathbf{X}$, j'utilise la notation classique $\mathbf{X}^{\mathbf{Y}}$. L'ensemble décrit ici est donc de la forme $\left(\mathbf{X}^{\mathbf{Y}}\right)^{\left(\mathbf{X}^{\mathbf{Y}}\right)}$ 
par rapport à la dimension $\mathbf{x}$ si et seulement si pour calculer sa valeur pour un argument donné, il ne faut tenir compte que de la valeur de l'argument dans les circonstances d'évaluation et non dans les circonstances qui diffèrent des circonstances selon la composante $\mathbf{x}$.

L'acceptation d'un tel critère a, entre autres, pour conséquence qu'un opérateur extensionnel par rapport à une ou plusieurs dimensions de circonstances d'évaluation, sera insensible à l'application de projecteurs sur son argument selon cette ou ces dimensions des circonstances d'évaluation. Plus précisément, si un opérateur O est extensionnel, alors la valeur de l'expression $\mathbf{O}\left(\downarrow_{\mathrm{x}} \mathbf{A}\right)$ dans une circonstance donnée pour cette circonstance sera la même que celle de l'expression $\mathbf{O}(\mathbf{A})$ dans les mêmes conditions.

Quelques exemples nous aideront à comprendre. Considérons l'énoncé «Le président des États-Unis est un homme de droite ». Selon notre critère, et en supposant que l'expression «actuel» signifie la même chose que «maintenant dans ce monde», nous pouvons tester que l'expression «est un homme de droite» est extensionnelle selon la composante temps et selon la composante monde possible parce que l'énoncé de départ a intuitivement la même valeur de vérité que l'énoncé «L'actuel président des ÉtatsUnis est un homme de droite $»$. Si nous refaisions le même exercice avec l'expression "était un homme de droite», nous arriverions à la conclusion que «était un homme de droite» n'est pas extensionnel par rapport au temps, ce qui était pour le moins prévisible. Tout cela est relativement trivial et est, à mon avis difficilement contestable.

Convenons maintenant d'appeler extensionnels (tout court) les opérateurs qui sont extensionnels pour toutes les composantes des circonstances d'évaluation. Remarquons que cela ne suppose pas que nous ayons identifié préalablement toutes les composantes pertinentes des circonstances d'évaluation. La valeur du test résultant de l'adoption de cette définition est susceptible de s'enrichir au fur et à mesure que la notion de circonstance elle-même s'enrichit. Remarquons également, et ceci est important en ce qui concerne la crédibilité de ma proposition, que les opérateurs tenus classiquement pour extensionnels résistent tous au test. 
La conjecture suivante est moins évidente. Selon le critère, le savoir et la croyance sont extensionnels. Plus précisément, si l'on accepte d'analyser les énoncés d'attitude propositionnelle comme exprimant une relation entre un locuteur et une signification, ici une intension, il est facile de se convaincre que si l'on tient pour vrai l'énoncé «aRp», l'on doit intuitivement tenir pour vrai l'énoncé « $\operatorname{aRf}(\mathrm{p})$ » où « $\mathrm{f}(\mathrm{p})$ » résulte d'une ou de plusieurs projections de $\mathbf{f}$ et $\mathbf{R}$ représente une attitude propositionnelle. Si Pierre croit qu'il pleut, il croit [qu'il pleut maintenant]'s. Si Pierre croit qu'il pleut, il croit qu'il pleut dans ce monde et non dans quelque monde bizarre compatible avec ses croyances. Bref, la conjecture est extrêmement générale et je défie qui que ce soit de trouver un contre-exemple.

Une objection très simple nous vient tout de suite à l'esprit. Il est certain que les attitudes propositionnelles de savoir et de croyance sont extensionnelles par rapport aux composantes déjà identifiées comme le temps, le monde possible, etc., ce qui nous manque est justement cette composante des circonstances d'évaluation à laquelle les opérateurs d'attitude propositionnelle sont sensibles. Il n'y a pas de telle composante, au moins en ce qui concerne le savoir et la croyance, et je vais essayer de montrer que c'est bien le cas.

\section{UN CRITĖRE EXTENSIONNEL DE SUBSTITUTION}

La dernière objection que j'ai soulevée pourrait être présentée de la façon suivante: la règle

savoir que $\mathrm{p}$ à $\left(\mathrm{c}_{1}, \ldots, \mathrm{c}_{\mathrm{m}}\right)$

savoir que $\left(\downarrow_{\mathrm{i}} \mathrm{p}\right)$ à $\left(\mathrm{c}_{1}, \ldots, \mathrm{c}_{\mathrm{m}}\right)$

5. J'utilise «[ «et»]» comme marqueur métalinguistique pour désambiguiser l'énoncé "Pierre croit qu'il pleut maintenant" qui pourrait se lire comme "Pierre croit maintenant qu'il pleut». Bien entendu, les opérateurs $O$ dont il est question sont à strictement parler intensionnels, en ce sens qu'ils prennent pour argument des intensions. Dire qu'ils sont extensionnels, signifie qu'ils ne sont sensibles qu'à la valeur de l'intension dans les circonstances de l'évaluation, bref à l'extension. Formellement $f\left(O\left(\downarrow_{x} A\right)\right)(c)$ est cette fonction $g$ telle que $g\left(c^{\prime}\right)=f(O(A))(c)\left(c^{\prime}\right)$ où $c^{\prime}$ est comme c' excepté qu'à la composante $x, c^{\prime}$ a la valeur de $c$. Pour que $O$ soit extensionnel, le critère dit que nous devons avoir

$$
f(O(A))(c)\left(c^{\prime}\right)=f(O(A))(c)\left(c^{\prime}\right)
$$

et c'est cette indifférence de $\mathrm{f}(\mathrm{O}(\mathrm{A}))(\mathrm{c})$ qui s'interprète intuitivement comme signe de l'extensionnalité. 
n'est valable que tant que « $\downarrow_{i}$ 》 ne touche à aucune des composantes des circonstances auxquelles justement le savoir est sensible. Il nous faut trouver un argument indépendant. En voici un. On m'accordera facilement, je crois, que la règle d'inférence suivante est intuitivement valide :

a sait que $\mathrm{p}$

a sait que la valeur de vérité de l'énoncé « $\mathrm{p}$ » est la même que la valeur de vérité de l'énoncé «q»

a sait que $\mathbf{q}$.

Ce critère de substituabilité ne nous apprend rien de lui-même. D'une part, la relation invoquée entre les contenus de savoir fait elle-même appel à une attitude propositionnelle, le savoir, et le critère tout entier est donc susceptible d'être accusé de circularité en démontrant ce qu'il présuppose. D'autre part, la présence de guillemets et l'utilisation du prédicat « valeur de vérité de » devraient nous inciter à la plus grande prudence.

Ce que je voudrais simplement faire remarquer, c'est qu'indépendamment de toute conception sur la nature des contextes de savoir et des problèmes que je viens d'évoquer, il est très difficile de maintenir que la signification de « la valeur de vérité de l'énoncé "p" est la valeur de vérité de l'énoncé "q" » dépend des significations de «p » et de « $\mathbf{q} »$. Dans le pire des cas, elle devrait dépendre de l'intension de « la valeur de vérité de l'énoncé » et des intensions de “"p" " et de «"q"». Autrement dit, pour que « savoir que p » entraîne «savoir que q", il suffit de savoir que la valeur de vérité de l'énoncé « $\mathbf{p}$ » est la même que la valeur de vérité de l'énoncé « $\mathbf{q}$ »dans les circonstances de l'évaluation. Cela est confirmé par le test développé plus haut: on peut projeter l'intension de «la valeur de vérité de l'énoncé "p" est la valeur de vérité de l'énoncé "q" » selon la composante que l'on voudra sans affecter la validité intuitive de la règle d'inférence.

Bref, nous avons toutes les raisons de croire que les intensions des énoncés qui sont les objets des attitudes n’interviennent en aucune façon dans les conditions de vérité (si ce n'est par leur extension, bien sûr). 


\section{LE PUZZLE DE KRIPKE}

Un bon argument allant à l'encontre de toute mon analyse serait qu'une théorie qui a pour conséquence que les attitudes propositionnelles sont extensionnelles, ne vaut pas grand'chose. Il me reste heureusement une échappatoire. La conclusion suivante que je tire de mon analyse est en fait beaucoup plus faible: en autant que la sémantique est concernée, les contextes de savoir (et de croyance) sont extensionnels. Plus précisément, ce qui fait problème avec les attitudes propositionnelles ne relève pas de la sémantique.

J'ai utilisé au début de mon intervention la métaphore du mode de donation objectif de la dénotation en parlant des significations, ce caractère objectif fixant d'une certaine manière la limite de la sémantique. Il est bien évident qu'en plus de ce mode de donation objectif de la dénotation, il existe un autre mode de donation - que l'on pourrait qualifier d'épistémique - de la dénotation. Comme la valeur de vérité des énoncés est absolument indépendante de la connaissance qu'a qui que ce soit de la signification des mots comme de la connaissance qu'a qui que ce soit des circonstances d'évaluation, le postulat de l'objectivité interdit que ce mode de donation épistémique soit d'une pertinence quelconque pour la sémantique à moins de pouvoir être intégré aux circonstances, ce qui, nous l'avons vu plus haut, pose des problèmes concernant le caractère finitaire des contenus sémantiques. Il nous faut alors, d'une manière ou d'une autre, sortir du champ de la sémantique, il nous faut élaborer une pragmatique des attitudes propositionnelles.

Remarquons que cela était déjà fortement suggéré par le fait que la règle présentée plus haut utilise des ressources qui sont du ressort de la pragmatique. On ne peut en effet que qualifier de pragmatique une relation qui fait intervenir des noms d'énoncés, leur valeur sémantique et des agents linguistiques.

Venons-en au puzzle de Kripke. Adapté au cadre que j'ai utilisé jusqu'ici, on peut le schématiser de la façon suivante. Si l'on autorise la substitution d'expressions de même signification dans des contextes de croyance, on est rapidement conduit à prêter à des locuteurs normaux et réfléchis des croyances qui ne sont que de grossières inconsistances. La connaissance ou plutôt l'absence de connaissance qu'ont les locuteurs des contenus sémantiques des 
expressions est extrêmement importante dans l'élaboration des critères de substitution. Ainsi que nous l'avons vu plus haut, cette connaissance est le point central de la question et ne porte en fait que sur les dénotations.

La voie de solution que je propose au puzzle de Kripke est simple. Bien sûr que les locuteurs normaux et réfléchis ont des croyances inconsistantes ; c'est même sur ces inconsistances potentielles que repose la logique de l'argumentation. N'est-ce pas l'émergence épistémique de l'inconsistance qui est susceptible d'amener un locuteur normal et réfléchi à changer ses croyances? Comment en est-on arrivé à la conclusion qu'Hesperus est bien Phosphorus après avoir longtemps cru le contraire? Dans quelle situation le fameux Pierre pourrait-il être amené à abandonner une de ses deux croyances?

Le véritable problème qui nous est posé par le puzzle de Kripke est bien plus de caractériser la notion de locuteur normal et réfléchi de sorte qu'elle n'interdise pas la possession de croyances inconsistantes, tout en restant compatible avec le cadre standard. Imaginons les conclusions pratiques que nous pourrions tirer de l'attitude du Pierre du puzzle. En s'appuyant sur sa rationalité et sur sa réflexion, nous pourrions tenter d'enrichir sa connaissance du monde jusqu'à ce qu'il soit impossible pour Pierre de continuer à soutenir que Londres n'est pas London.

J'ai proposé ailleurs une telle caractérisation mais elle est un peu trop technique pour la reprendre ici. Je vais simplement en présenter les grandes lignes. Un locuteur normal et réfléchi est un locuteur qui ne sait rien de faux et qui manie les constantes logiques de façon acceptable. Prenant ces seules contraintes comme point de départ, il s'agit alors de caractériser ces «espaces de savoir » d'agents normaux et réfléchis. Cela conduit à la définition d'interprétations idiosyncratiques respectant les contraintes en question. La seule restriction sur ces valeurs sémantiques idiosyncratiques qu'un locuteur normal et réfléchi attribue aux expressions de son langage est qu'elles ne peuvent l'induire en erreur, au sens ou ce locuteur serait amené à savoir quelque chose de faux. Formellement, ces interprétations idiosyncratiques peuvent se représenter comme des fonctions qui attribuent des valeurs sémantiques partielles aux expressions du langage du locuteur et la 
contrainte de normalité s'exprime dans le fait que lorsque ces valeurs sémantiques partielles sont définies, leur contribution aux valeurs sémantiques des énoncés où elles apparaissent ne peut conduire à attribuer à ces énoncés la mauvaise valeur de vérité.

Un locuteur réfléchi tire les conséquencs logiques de tout ce qu'il sait, mais cela ne constitue qu'une partie des énoncés vrais de son langage. L'idée sous-jacente est, encore une fois pour reprendre ma métaphore du début, que le mode de donation du contenu épistémique n'a de pertinence dans la compréhension du langage et donc dans la communication qu'en autant qu'il constitue une approximation de ce mode de donation objectif postulé au départ.

Concluons. Croire que p c'est s'engager à ce que $\mathrm{p}$ soit vrai. En autant que la sémantique est concernée, c'est s'engager envers le vrai ou le faux. Le fait que cet engagement se fasse à travers $\mathrm{p}$ ne relève pas exclusivement de la sémantique mais de la pragmatique. Par contre, les règles de ce jeu pragmarique doivent dans une certaine mesure être compatible avec l'interprétation objective visée car après tout, ce jeu se veut pertinent pour la communication. Un locuteur normal et réfléchi peut s'engager à ce que deux énoncés incompatibles (et même nécessairement incompatibles comme dans le cas de Pierre) soient vrais en autant que cela ne le contraigne pas à savoir quelque chose de faux. Ma thèse est simplement que cette contrainte constitue une base suffisante pour l'élaboration d'une logique du savoir et de là des attitudes propositionnelles en général.

Département de philosophie, Université de Montréal 